Virginia Commonwealth University VCU Scholars Compass

1982

\title{
Effective field model for Ising ferromagnets: Influence of triplet correlations
}

G. B. Taggart

Virginia Commonwealth University

Follow this and additional works at: http://scholarscompass.vcu.edu/phys_pubs

Part of the Physics Commons

Taggart, G. B. Effective field model for Ising ferromagnets: Influence of triplet correlations. Journal of Applied Physics 53, 1907 (1982). Copyright (C) 1982 AIP Publishing LLC.

\section{Downloaded from}

http://scholarscompass.vcu.edu/phys_pubs/138

This Article is brought to you for free and open access by the Dept. of Physics at VCU Scholars Compass. It has been accepted for inclusion in Physics Publications by an authorized administrator of VCU Scholars Compass. For more information, please contact libcompass@vcu.edu. 


\title{
Effective field model for Ising ferromagnets: Influence of triplet correlations ${ }^{\text {a) }}$
}

\author{
G. B. Taggart \\ Department of Physics, Virginia Commonwealth University, Richmond, Vinginia 23284
}

We use an effective field model which explicitly incorporates correlations to determine the critical temperatures for Ising ferromagnets on the common lattices. We specifically retain only the first nontrivial odd correlation function, i.e., the triplet correlation function, while neglecting higher order correlations. Within this approximation, assuming the equivalence of triplet correlations involving both central and noncentral sites in the nearest neighbor cluster yields values for the critical temperature below the Bethe approximations for $z \geq 4$. Likewise, assuming the inequivalence of triplet correlations involving the central and noncentral sites yields values of the critical temperature only slightly higher than the Bethe approximation for $z \geq 4$. For lattices with four- and six-fold coordination this equivalence and inequivalence of triplet correlation functions is sufficient to cause the critical temperatures to approach their two- and threedimensional values. For instance, for quadratic lattices we obtain values for the critical temperature of 2.5284 and 2.9796 as compared to the exact and series results of 2.2692 and 2.7044 for the simple quadratic and diamond lattices, respectively, and the Bethe approximation of 2.8854 .

PACS numbers: $75.10 . \mathrm{Hk}, 05.50 .+\mathrm{q}$

There have recently been a number of publications dealing with new approximation schemes for the spin-tis Ising mode1 (1-5). These approximations share common elements (6); one of these belng the use of rigorous correlation identities as a starting point. In the critical region values of the transition temperature calculated from these effective field models are improved markedly over the usual mean field approximation (MFA) and most other cluster, or closed-form, approximations, but they are not as accurate as those determined from renormalization group methods.

We have recently developed another varlant of these approximation schemes (7) which utilizes two sets of correlation identites rather than the one set used in previous work. Applying relatively simple approximations to these identities we are able to readily improve on the values of the critical temperature determined by the Bethe approximation. The approximation scheme explicitly takes into account the effects of correlations.

As is well known, most effective field models are not able to distinguish between lattices having common numbers of nearest neighbors but different dimensionalities e.g., the simple quadratic lattice (SQL) and the diamond lattice (DL). The cluster variation method of Kikuchi is one method which can do this (8), and in the spirit of this paper, the approach of Matsudaira has this ability. The intent of the present work is to determine if we can use the two sets of correlation identities as a basis for an approximation scheme which can distinguish between different lattices with common numbers of nearest neighbors.

\section{CORRELATION IDENTITIES}

of the various approximation schemes mentioned in the introduction that of Honmura and Kaneyoshi (HK) (2) is of particular interest here. HK used an exponential operator formalism to rewrite the standard Ising identity (10).

$$
\left\langle\sigma_{g}\{g\}\right\rangle=\left\langle\{g\} \tanh \beta \sum_{p} J_{g p} \sigma p,\right.
$$

as,

$$
\begin{array}{r}
\left.<\sigma_{g}\{g\}\right\rangle=\left\langle\{g\} \prod_{p}\left(\cosh D B J_{g p}+\sigma_{p} \sinh D \beta J_{g p}\right) .\right. \\
\left.\cdot \tanh x\right|_{x=0} .
\end{array}
$$

Here $\sigma_{g}$ are the Ising variables $\left(\sigma_{g}= \pm 1\right)$ and the brac- kets represent the usual thermal average under the Ising Hamiltonian,

$$
H=-\frac{1}{2} \sum_{g, p} J_{g p} \sigma_{g} \sigma_{p} .
$$

$\{g\}$ represents any function of the Ising variables as long as it is a function of $\sigma_{p}(p \neq g)$ and the differential operator is,

$$
D \equiv \frac{\partial}{\partial x}
$$

By restricting the effective interactions $\mathrm{J}$ to the $z$ nearest neighbors about the central site $g \equiv 0$ we can rewrite eqn. (2) as,

$$
\begin{aligned}
&\left\langle\sigma_{0}\{g\}\right\rangle=\left\langle\{g\} \prod_{p=1}^{\mathrm{z}}(\cosh \mathrm{DK}+\right.\left.\left.\sigma_{\mathrm{p}} \sinh \mathrm{DK}\right)\right\rangle \cdot \\
&\left.\cdot \tanh x\right|_{\mathrm{x}=0}
\end{aligned}
$$

where $K \equiv \beta J$. For the case when $z=4$, for instance, we set $\{g\}=1$ and expand eqn. (5),

$$
\begin{gathered}
<\sigma_{0}>\equiv \mathrm{m}=\left\langle\sigma_{1}+\sigma_{2}+\sigma_{3}+\sigma_{4}>\mathrm{A}_{4}(1)\right. \\
+<\sigma_{1} \sigma_{2} \sigma_{3}+\sigma_{1} \sigma_{3} \sigma_{4}+\sigma_{2} \sigma_{3} \sigma_{4}+\sigma_{1} \sigma_{2} \sigma_{4}>A_{4}(3),
\end{gathered}
$$

where $A_{z}(n)$ is the coefficient of the n-site correlation function, or,

$$
A_{4}(1)=\frac{1}{8}(\tanh 4 K+2 \tanh 2 K),
$$

and

$$
\mathrm{A}_{4}(3)=\frac{1}{8}(\tanh 4 \mathrm{~K}-2 \tanh 2 \mathrm{~K}) \text {. }
$$

By assuming the statistical independence of lattice sites we can write eqn. (6) as,

$$
\mathrm{m}=4 \mathrm{~m} \mathrm{~A}_{4}(1)+4 \mathrm{~m}^{3} \mathrm{~A}_{4}(3) \text {. }
$$

This expression then yields the magnetization curve for quadratic lattices. As the magnetization becomes vanishingly small eqn. (8) can be linearized to yield the critical condition,

$$
1=4 \mathrm{~A}_{4}^{\mathrm{C}}(1) \text {, }
$$

and the root,

$$
\mathrm{K}_{\mathrm{c}}^{-1}=3.0898
$$


This value for $\mathrm{K}_{\mathrm{c}}^{-1}$ is an improvement on the usual MFA of 4.0 , but not on the Bethe approximation of 2.8854 .

Taggart and Fittipaldi (7) made use of a second set of correlation identities for the Ising model (10),

$$
\langle\{g\}\rangle=\left\langle\sigma_{g}\{g\} \operatorname{coth} \beta \sum_{p} J_{g p} \sigma_{p}\right\rangle
$$

which is formally the inverse of eqn. (1). Following the procedure of HK we can write,

$$
\begin{aligned}
& \langle\{g\}\rangle=\left\langle\sigma_{0}\{g\} p_{p^{\frac{Z}{I}}}^{\frac{Z}{1}}\left(\operatorname{coth} D K+\sigma_{p} \sinh D K\right)\right\rangle \cdot \\
& \text { - }\left.\operatorname{coth} x\right|_{x=0} \text {. }
\end{aligned}
$$

Expanding this expression we obtain,

$$
\begin{gathered}
\langle\{g\}\rangle=\left\langle\sigma_{0}\{g\}\left(\sigma_{1}+\sigma_{2}+\sigma_{3}+\sigma_{4}\right)>\tilde{A}_{4}(1)\right. \\
+\left\langle\sigma_{0}\{g\}\left(\sigma_{1} \sigma_{2} \sigma_{3}+\sigma_{1} \sigma_{3} \sigma_{4}+\sigma_{2} \sigma_{3} \sigma_{4}+\sigma_{1} \sigma_{2} \sigma_{4}\right)>\tilde{A}_{4}(3),\right.
\end{gathered}
$$

where $\widetilde{A}_{z}(n)$ is obtained from $A_{z}(n)$ by replacing tanh $x$ by $\operatorname{coth} \mathrm{x}$. When $\{\mathrm{g}\}=1$ eqn. (13) gives a relationship between the pair and quadruplet correlations. If we assume that $\{\mathrm{g}\}=\sigma_{1}$, say, and assume statistical independence, then eqn. (13) yields a critical temperature worse than the MFA. However Taggart and Fittipaldi were able to use eqns. (5) and (12) consistently to yield estimates of $\mathrm{K}_{\mathrm{c}}^{-1}$ better than the

Bethe approximation.

\section{TRIPLET APPROXIMATION}

As with most simple effective field approximations we cannot distinguish between lattices such as the SQL and DL which have the same number of nearest neighbors but differ in dimensionality. The effective field models which do make this distinction, i.c., the cluster variation method or Matsudaira's higher order approximations, do so by making assumptions about the influence and magnitudes of higher order correlations. We will follow that same spirit here.

We will use the quadratic lattice as an example and rewrite eqn. (6) as,

$$
m=4 \mathrm{~mA}_{4}(1)+4 \tau \mathrm{A}_{4}(3),
$$

where $\tau \equiv\left\langle\sigma_{i} \sigma_{j} \sigma_{k}\right\rangle, i \neq j \neq k=1-4$, is the triplet correlation not referring to the central site. This is an exact relationship. Now eqn. (13) can be rewritten (with $\{\mathrm{g}\}=\sigma_{1}$ ) as,

$m=m \tilde{A}_{4}(1)+3 \tau_{0}\left(\tilde{A}_{4}(1)+\tilde{A}_{4}(3)\right)+(1) \tilde{A}_{4}(3)$,

where $\tau_{0} \equiv\left\langle\sigma_{0} \sigma_{i} \sigma_{j}\right\rangle, i \neq j \neq 1-4$ is the triplet correlation with reference to the central site and $\omega_{0}$ is the five-site correlation. This is also an exact relationship.

In order to solve these equations near the critical point we neglect the fifth-order correlation in eqn. (15), and propose that $\tau \cong \tau_{0}$-for the SQL and $\tau \neq \tau_{0}$ for the DL. This assumption is in the same spirit as those inherent in the cluster variation method and the approximations of Matsudaira. Thus we can now solve eqns. (14) and (15) at the critical point for the SQL, while for the DL we need another equation. This we obtain by setting $\{\mathrm{g}\}=\sigma_{1} \sigma_{2}$ in eqn. (5) to get,

$$
\tau_{0}=2(m+\tau)\left(A_{4}(1)+A_{4}(3)\right) .
$$

Solving eqns. (14) and (15) for the SQL we find the root $\mathrm{K}_{\mathrm{c}}^{-1}=2.5284$, as compared to the exact root of 2.2692 and that of the Bethe approximation of 2.8854. Similarly, solving eqns. (14) - (16) for the DL we get the root $\mathrm{K}_{\mathrm{c}}^{-1}=2.9796$ versus the series result of 2.7044 . We have solved similar equations for the critical temperatures for all common lattices and the results are given in Table $I$.

\section{DISCUSSION}

A number of points can be made concerning the results presented in Table I. First, as $z$ increases, in this triplet approximation, correlation functions of higher order are neglected. Thus the results for $z=3$ are more "exact" than those for $z=12$ and more sensitive to any approximations made. Second, as $z$ in-

\begin{tabular}{|c|c|c|c|c|c|}
\hline$z$ & 3 & 4 & $6^{\text {b) }}$ & 8 & 12 \\
\hline $\mathrm{HK}$ & 2.1037 & 3.0898 & 5.0733 & 7.0606 & 11.0445 \\
\hline Bethe & 1.8205 & 2.8854 & 4.9326 & 6.9521 & 10.9696 \\
\hline Here $\left(\tau=\tau_{0}\right)$ & -- & 2.5284 (SQL) & $4.4193(\mathrm{PT})$ & 6.3717 & 10.3290 \\
\hline Here $\left(\tau \neq \tau_{0}\right)$ & 3.4913 & 2.9796 (DL) & $4.9924(\mathrm{SC})$ & 6.9959 & 10.9983 \\
\hline Matsudaira & -- & 2.622 (SQL) & $\begin{array}{l}4.271 \text { (PT) } \\
4.731 \text { (SC) }\end{array}$ & 6.663 & -- \\
\hline Exact/Series & 1.5186 & $\begin{array}{ll}2.2692 & (\mathrm{SQL}) \\
2.7044 & \text { (DL) }\end{array}$ & $\begin{array}{l}3.6410 \text { (PT) } \\
4.5108 \text { (SC) }\end{array}$ & 6.3533 & 9.7952 \\
\hline
\end{tabular}
creases values of $\mathrm{K}_{\mathrm{c}}^{-1}$ for $\tau=\tau_{0}$ and $\tau \neq \tau_{0}$ approach or go above the series values. This would indicate that the distinction, or similarity, between $\tau$ and $\tau_{0}$ is

not as critical at higher coordination number as with lower. Also, in light of our first comment, there is

Table I 
also more of an averaging effect at higher $z$ due to neglect of higher order correlations.

This simple assumption concerning the influence of triplet correlations has been sufficient to produce a distinction between lattices having the same numbers of nearest neighbors but of different dimensionality. Though the method is somewhat ad hoc it is in the same spirit as other effective field models which have this ability and, as with these, is justiflable a posteriori. The approximation can be readily improved by including higher order correlations, as well as by introducing decoupling approximations. In addition, correlation functions can be readily calculated from the system of equations.

\section{REFERENCES}

a) Supported by the Grants-in-Aid Program for Faculty of Virginia Commonwealth University. b) Plane triangle (PT) and simple cubic (SC).

1) B. Frank and O. Mitran, J. Phys. C: Solid St. Phys. 10, 2641 (1977); ibid. 11, 2087 (1978).

2) R. Honmura and $T$. Kaneyosh1, Prog. Theor. Phys. 60,635 (1978); J. Phys. C: Sol1d St. Phys, 12, $\overline{39} 79$ (1979).

3) D. C. Mattis, Phys, Rev, B 19,4737 (1979).

4) H. I. Zhang, J. Phys, C: Solid St. Phys. 14, 57 (1981).

5) T. Kaneyoshi, I. P. Fittipaldi, R. Honmura, and T. Manabe, Phys. Rev. B 24, 481 (1981).

6) H. I. Zhang and D. Kim, Phys. Rev. B 21, 4173 (1980); T. Kaneyoshi, Phys. Letters $\overline{76 A}, 67$ (1980).

7) G. B. Taggart and I. P. Fittipaldi, $\overline{B u I}$. Am. Phys. Soc. 26,380 (1981); (to be published).

8) D. M. Burley in Phase Transitions and Critical Phenomena, edited by C. Domb and M. S. Green, Vol. 2, Academic Press, NY, 1972, Ch. 9.

9) N. Matsudaira, J. Phys. Soc. Jpn. 35, 1593 (1973).

10) H. B. Callen, Phys. Letters 4,161 (1963). 